\title{
JAK2 inhibitor blocks the inflammation and growth of esophageal squamous cell carcinoma in vitro through the JAK/STAT3 pathway
}

\author{
JUEMIN FANG, LI CHU, CHUNYAN LI, YIJING CHEN, FEI HU, XI ZHANG, \\ HUAXIN ZHAO, ZHUQING LIU and QING XU \\ Department of Medical Oncology, Shanghai Tenth People's Hospital, Tongji University, \\ School of Medicine, Shanghai 200072, P.R. China
}

Received September 22, 2014; Accepted October 23, 2014

DOI: 10.3892/or.2014.3609

\begin{abstract}
Recent research indicates that the Janus kinase/signal transducer and activator of transcription 3 (JAK/STAT3) pathway may play an important role in chronic inflammation which promotes cancer progression, yet the mechanism is not clear. The present study aimed to investigate the role of the JAK/STAT3 pathway in the growth and cancer-related inflammation (CRI) of esophageal squamous cell carcinoma (ESCC) by studying the crosstalk between the JAK/STAT3 pathway and nuclear factor- $\kappa \mathrm{B}(\mathrm{NF}-\kappa \mathrm{B})$ and cyclooxygenase-2 (COX-2) which are important inflammatory factors associated with tumorigenesis. Cell growth and the cell cycle were assessed by CCK-8 assays and flow cytometry, respectively. The protein levels of STAT3, phosphorylated STAT3, VEGF, $\mathrm{NF}-\kappa \mathrm{B}$ p65, phosphorylated NF- $\kappa \mathrm{B}$ p65 and COX-2 in ESCC cells following treatment with JAK2 inhibitor for $48 \mathrm{~h}$ or interleukin-6 (IL-6) for $24 \mathrm{~h}$ were detected. RT-PCR was performed to study the interaction among STAT3, NF- $\mathrm{KB}$ and COX-2 by transfection of siRNAs targeted at STAT3 and NF- $\kappa$ B. STAT3 was activated in 3 ESCC cell lines at different levels. Blocking the JAK/STAT3 pathway inhibited cancer growth through regulation of cell growth, cell cycle and angiogenesis. Likewise, abrogation of the JAK/STAT3 pathway decreased $\mathrm{CRI}$ by downregulating levels of $\mathrm{NF}-\kappa \mathrm{B}$ p 65 phosphorylation, COX-2 and IL- 6 concentration. In addition, CRI and cancer growth were accelerated by IL- 6 through stimulation of the JAK/STAT3 and NF- $\kappa$ B p65 pathway. Moreover, STAT3 and $\mathrm{NF}-\kappa \mathrm{B}$ both regulated COX-2 as a downstream gene. The JAK/STAT3 pathway is an important pathway which links CRI and cancer growth through IL-6 and crosstalk with the $\mathrm{NF}-\kappa \mathrm{B}$ p65 subunit and COX-2. The STAT3 pathway could
\end{abstract}

Correspondence to: Professor Qing Xu, Department of Medical Oncology, Shanghai Tenth People's Hospital, Tongji University, School of Medicine, 301 Yanchang Road, Shanghai 200072, P.R. China

E-mail: xuqingmd@gmail.com

Key words: JAK/STAT3 pathway, esophageal squamous cell carcinoma, cancer-related inflammation, NF- $\kappa$ B, JAK2 inhibitor be a novel target both for cancer treatment and prevention in ESCC.

\section{Introduction}

Esophageal squamous cell carcinoma (ESCC) is one of the most common and aggressive cancers in the world, particularly with high incidence and morbidity in China (1,2). Although treatment strategies have progressed in recent years, the prognosis of ESCC is still poor. It is urgent to identify a new target to improve patient outcomes. Signaling pathways have been widely studied in cancers; however, the precise mechanisms underlying ESCC are poorly understood.

Recently, cancer-related inflammation (CRI) has been considered as the seventh hallmark of cancer (3). A close relationship has been revealed between chronic inflammatory infection and cancer risk and progression such as Helicobacter pylori and gastric cancer, papilloma virus and cervical cancer, and hepatitis viruses and liver carcinoma. Human papilloma virus (HPV) infection has also been suggested as an etiology of ESCC, in particular types 16 and 18, although the conclusion is controversial $(4,5)$. In addition, overexpression of several inflammatory markers, cyclooxygenase-2 (COX-2) and nuclear factor $-\kappa \mathrm{B}(\mathrm{NF}-\kappa \mathrm{B})$, have also been observed in ESCC with predictive prognostic value, indicating the inflammatory mechanisms in ESCC development (6). However, the relationship between chronic inflammation and ESCC is also poorly understood.

More and more evidence indicates that various molecular and cellular pathways are involved in the link between inflammation and cancer (7). Among them, the signal transducer and activator of transcription 3 (STAT3) and NF- $\mathrm{B}$ signaling pathways are considered to play a vital role in regulating inflammation and cancer development (8). Persistent abnormal activation of STAT3 is oncogenic in many human cancers, including breast, prostate, ovarian cancers, and pancreatic cancer (9-13). Activated STAT3 promotes carcinogenesis through regulation of downstream genes that encode cell apoptosis, cell cycle, metastasis and angiogenesis $(14,15)$. Janus kinase (JAK) is responsible for STAT3 activation when stimulated by extracellular signals, and the JAK2 inhibitor 
AG490 was found to block the constitutive activation of STAT3 (16). Recently, a new role of STAT3 in CRI has been reported by promoting pro-oncogenic inflammatory pathways through the IL-6-GP130-JAK pathways, and also by opposing antitumor immune responses while the underlying mechanism is not fully clarified (17). Interleukin-6 (IL-6) belongs to a large family of cytokines and activates JAKs through binding to its receptor and dimerizing glycoprotein 130 (gp130) which is expressed on many cells (18). It is involved in many inflammatory processes and promotes oncogenesis. Recently, a colitis-associated cancer model confirmed that IL- 6 and STAT3 are essential in the process from chronic inflammation to cancer. Nevertheless, studies focusing on the effects of STAT3 on ESCC are few compared with other cancers and their roles in ESCC CRI and cancer growth are unknown (19).

$\mathrm{NF}-\kappa \mathrm{B}$ is another important regulator abnormally activated in CRI in many types of cancers, and it also can be triggered by IL-6 (20). When activated, NF- $\kappa \mathrm{B}$ induces the generation of many molecules modulating inflammation, angiogenesis and adhesion. In addition, it was found to participate in CRI to promote tumor initiation and progression in a liver and gastrointestinal tract cancer model $(21,22)$. The NF- $\kappa \mathrm{B}$ family

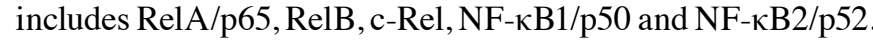
Among them, RelA/p65 is reported to be closely correlated with inflammation, cell proliferation, survival signals and cancer. However, the crosstalk between the STAT3 and NF- $\mathrm{BB}$ signaling pathways in CRI and oncogenesis has not been fully elucidated.

In the present study, expression levels of STAT3 and its phosphorylated form in human ESCC cell lines were examined. Additionally, the interrelationship between the STAT3

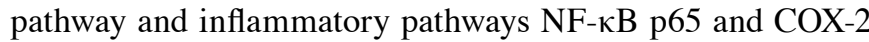
were preliminarily studied. Our objective was to demonstrate that the STAT3 signaling pathway may be a key regulator linking cancer growth and CRI in ESCC. Inhibition of this pathway may be a novel treatment and preventative target for ESCC.

\section{Materials and methods}

Cell lines and reagents. The human ESCC cell line TE-1 (catalog no. TCHu 89) was obtained from the Cell Bank of Shanghai Institute (Shanghai, China). Cell lines EC-1 and K150 were provided by Professor Fenyong Sun, Department of Central Laboratory, Shanghai Tenth People's Hospital. The cells were maintained in RPMI-1640 containing $10 \%$ fetal bovine serum and $100 \mathrm{U} / \mathrm{ml}$ penicillin and $100 \mu \mathrm{g} / \mathrm{ml}$ streptomycin. All cells were incubated at $37^{\circ} \mathrm{C}$ in a humidified atmosphere containing $5 \% \mathrm{CO}_{2}$. AG490 was purchased from Merck (Whitehouse Station, NJ, USA), dissolved in dimethyl sulfoxide and then diluted with the culture medium for experiments. Recombinant IL-6 was purchased from PeproTech (Princeton, NJ, USA), dissolved in acetic acid and then diluted with the culture medium. Monoclonal antibodies against $\beta$-actin were purchased from Sigma-Aldrich (St. Louis, MO, USA). Primary antibodies against STAT3 and phosphoSTAT3 (Tyr705), COX-2, NF- $\mathrm{B}$ p65 for western blotting were purchased from Cell Signaling Technology (Danvers, MA, USA) and p-NF- $\kappa$ B p65 (Ser536) was from Santa Cruz Biotechnology (Santa Cruz, CA, USA). Human IL-6 ELISA kit was purchased from R\&D Systems (Minneapolis, MN, USA).

Cell proliferation assay. Cell proliferation and viability were determined by the Cell Counting Kit-8 (CCK-8) colorimetric assay (Dojindo, Japan). TE-1 and EC-1 cells were seeded in a 96-well plate at $3 \times 10^{3}$ cells/well. When the cell density reached $80 \%$, fresh culture medium containing AG490 (5, 10, 20, 40 and $80 \mu \mathrm{mol} / \mathrm{l}$ ) was added to the cells after $12 \mathrm{~h}$ of starvation, respectively. Cell viability was measured using the CCK-8 assay after culture for 24 and $48 \mathrm{~h}$. Absorbance was measured at $450 \mathrm{~nm}$ with a microtiter plate reader.

Flow cytometry for cell cycle analysis. TE-1 cells treated with AG490 at different concentrations for $48 \mathrm{~h}$ were washed and fixed with phosphate-buffered saline (PBS) for two times and then fixed with ethanol $95 \%$ and washed with cold PBS and then resuspended in $150 \mu \mathrm{l}$ hypotonic fluorochrome solution [50 $\mu \mathrm{g} / \mathrm{ml}$ propidium iodide (PI), $10 \mu \mathrm{g} / \mathrm{ml}$ RNAse A in PBS]. The cells were incubated in the dark at $4^{\circ} \mathrm{C}$ overnight before flow cytometric analysis was performed. The PI fluorescence of individual nuclei was measured using a FACSCalibur cytometer (BD Biosciences, Heidelberg, Germany). Data were analyzed with the CellQuest Pro v 5.2.1 software (BD Biosciences). For each condition, at least 3 independent experiments were performed.

Western blotting. TE-1 cells were treated with AG490 (5, 10, 20,40 and $80 \mu \mathrm{mol} / \mathrm{l}$ ) for $48 \mathrm{~h}$, respectively. EC-1 cells were treated with $100 \mathrm{ng} / \mathrm{ml}$ IL- 6 for $24 \mathrm{~h}$. The harvested cells were washed with PBS twice and lysed on ice for $30 \mathrm{~min}$ with whole cell extract lysis buffer (Santa Cruz Biotechnology). Lysates were centrifugated at $12,000 \mathrm{rpm}$ for $10 \mathrm{~min}$ at $4^{\circ} \mathrm{C}$, and the protein concentration was determined by an assay kit (Bio-Rad, Hercules, CA, USA). Cell lysates were mixed with loading buffer and boiled for $5 \mathrm{~min}$ at $100^{\circ} \mathrm{C}$. Protein samples were separated by sodium dodecyl sulfate-polyacrylamide gel electrophoresis and transferred onto nitrocellulose membranes. The membranes were blocked in blocking buffer (Tris-buffered saline, $0.1 \%$ Tween-20 and 5\% non-fat dry milk) for $1 \mathrm{~h}$ and then incubated overnight at $4^{\circ} \mathrm{C}$ with the specific anti-STAT3 antibody (1:1,000 dilution), anti-p-STAT3 antibody (1:1,000 dilution), anti-NF- $\mathrm{B}$ p65 antibody (1:500 dilution), anti-pNF- $\mathrm{B}$ p65 antibody (1:500 dilution), anti-vascular endothelial growth factor (VEGF) antibody (1:1,000 dilution), anti-COX-2 antibody (1:500 dilution) and anti- $\beta$-actin antibody $(1: 2,000$ dilution). Subsequently, the membranes were incubated with a horseradish peroxidase-conjugated secondary antibody rabbit IgG (1:2,000 dilution; Santa Cruz Biotechnology) for $1 \mathrm{~h}$ at room temperature after being washed in TBS/0.1\% Tween-20 for 3 times. Then after 3 washes in TBS/0.1\% Tween-20 again, the membranes were detected by chemiluminescence using Western Blotting Luminol Reagent (Santa Cruz Biotechnology).

$R N A$ transfection and RT-PCR. Cells were transfected with a non-specific random siRNA as a negative control and synthetic siRNA (GenePharma, Shanghai, China) directed to STAT3 or NF- $\mathrm{B}$ p65 (3 siRNAs for each gene) at a final concentration of $100 \mathrm{nM}$ for $48 \mathrm{~h}$. Total RNA was extracted by TRIzol LS 
Table I. Inhibitory effect of AG490 on TE-1 cell growth.

\begin{tabular}{lcccc}
\hline & \multicolumn{2}{c}{$\mathrm{A}_{450}(\mathrm{mean} \pm \mathrm{SD})$} & \multicolumn{2}{c}{ Inhibition rate $(\%)$} \\
\cline { 2 - 5 } Group & $24 \mathrm{~h}$ & $48 \mathrm{~h}$ & $24 \mathrm{~h}$ \\
\hline Blank control & $0.876 \pm 0.004$ & $1.465 \pm 0.010$ & 0 & 0 \\
$5 \mu \mathrm{mol} / \mathrm{l} \mathrm{AG} 490$ & $0.847 \pm 0.007$ & $1.261 \pm 0.018$ & 3.31 \\
$10 \mu \mathrm{mol} / \mathrm{l} \mathrm{AG} 490$ & $0.839 \pm 0.006$ & $1.249 \pm 0.008$ & $4.22^{\mathrm{a}}$ \\
$20 \mu \mathrm{mol} / \mathrm{l} \mathrm{AG} 490$ & $0.768 \pm 0.005$ & $1.168 \pm 0.005$ & $12.33^{\mathrm{b}}$ & $13.92^{\mathrm{b}}$ \\
$40 \mu \mathrm{mol} / \mathrm{l} \mathrm{AG} 490$ & $0.753 \pm 0.007$ & $1.157 \pm 0.038$ & $14.04^{\mathrm{b}}$ & $20.27^{\mathrm{b}}$ \\
$80 \mu \mathrm{mol} / \mathrm{l} \mathrm{AG} 490$ & $0.652 \pm 0.004$ & $1.026 \pm 0.046$ & $25.57^{\mathrm{b}}$ & $21.02^{\mathrm{b}}$ \\
\hline
\end{tabular}

${ }^{\mathrm{a}} \mathrm{P}<0.05,{ }^{\mathrm{b}} \mathrm{P}<0.001$ vs. blank control group. AG490, JAK2 inhibitor.

Table II. Inhibitory effect of AG490 on EC-1 cell growth.

\begin{tabular}{|c|c|c|c|c|}
\hline \multirow[b]{2}{*}{ Group } & \multicolumn{2}{|c|}{$\mathrm{A}_{450}($ mean $\pm \mathrm{SD})$} & \multicolumn{2}{|c|}{ Inhibition rate $(\%)$} \\
\hline & $24 \mathrm{~h}$ & $48 \mathrm{~h}$ & $24 \mathrm{~h}$ & $48 \mathrm{~h}$ \\
\hline Blank control & $0.774 \pm 0.007$ & $1.827 \pm 0.057$ & 0 & 0 \\
\hline $5 \mu \mathrm{mol} / \mathrm{l} \mathrm{AG} 490$ & $0.752 \pm 0.006$ & $1.755 \pm 0.019$ & 2.84 & $3.94^{\mathrm{a}}$ \\
\hline $10 \mu \mathrm{mol} / \mathrm{l} \mathrm{AG} 490$ & $0.713 \pm 0.008$ & $1.673 \pm 0.036$ & $7.88^{\mathrm{a}}$ & $8.43^{\mathrm{b}}$ \\
\hline $20 \mu \mathrm{mol} / \mathrm{l} \mathrm{AG} 490$ & $0.675 \pm 0.007$ & $1.585 \pm 0.046$ & $12.79^{b}$ & $13.25^{\mathrm{b}}$ \\
\hline $40 \mu \mathrm{mol} / \mathrm{l} \mathrm{AG} 490$ & $0.657 \pm 0.006$ & $1.519 \pm 0.012$ & $15.11^{\mathrm{b}}$ & $16.86^{\mathrm{b}}$ \\
\hline $80 \mu \mathrm{mol} / \mathrm{l} \mathrm{AG} 490$ & $0.573 \pm 0.002$ & $1.315 \pm 0.054$ & $25.97^{\mathrm{b}}$ & $28.02^{\mathrm{b}}$ \\
\hline
\end{tabular}

${ }^{\mathrm{a}} \mathrm{P}<0.05,{ }^{\mathrm{b}} \mathrm{P}<0.001$ vs. blank control group. AG490, JAK2 inhibitor.

(Invitrogen, Carlsbad, CA, USA). A spectrophotometer was used to detect the concentration and purity of the RNA. cDNA was synthesized with reverse transcriptase (Takara, Japan). Quantitative real-time polymerase chain reaction (RT-PCR) assays were carried out on the ABI Prism 7500 Fast System (Applied Biosystems, Carlsbad, CA, USA) using the standard curve method. The following primers were used: STAT3 sense strand, 5'-AGCATCCTGAAGCTGACCCAGGT-3' and antisense strand, 5'-TCGGCAGGTCAATGGTATTGCTGC-3'; NF- $\kappa$ B p65 sense strand, 5'-TCTGCTTCCAGGTGACAGTG-3' and antisense strand, 5'-ATCTTGAGCTCGGCAGTGTT-3'; COX-2 sense strand, 5'-CCCCCACAGTCAAAGACACT-3' and antisense strand, 5'-CTCATCACCCCACTCAGGAT-3'.

\section{Results}

STAT3 is phosphorylated in human ESCC cell lines. STAT3 phosphorylation and COX-2 upregulation are closely related to cancer development and CRI. In the present study, we measured the protein levels of STAT3, phosphorylated STAT3 as well as COX-2 by performing western blotting in 3 human ESCC cell lines EC-1, TE-1 and K150. STAT3 was activated in the 3 cell lines at different levels. STAT3 phosphorylation was highest in the TE-1 and lowest in the EC-1 cells. COX-2 was also expressed in the TE-1 and K150 cells but very low in the EC-1 cells (Fig. 1).

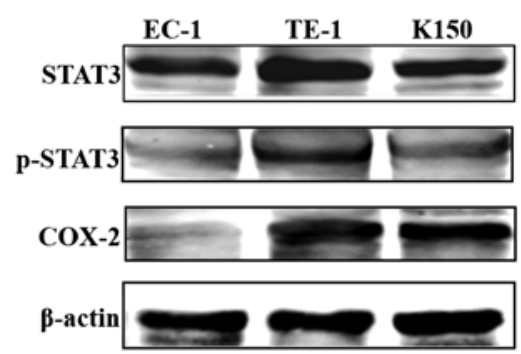

Figure 1. Protein levels of total STAT3, STAT3 phosphorylation and COX-2 by western blotting. STAT3, signal transducer and activator of transcription 3; COX-2, cyclooxygenase-2.

JAK2 inhibitor attenuates the growth of ESCC by regulating cell growth and cell cycle. A positive correlation between STAT3 activation and cancer cell growth has been verified. In to the present study, cell proliferation assay was conducted with TE-1 and EC-1 cells treated with AG490 at different concentrations for 24 and $48 \mathrm{~h}$. As expected, AG490 reduced the proliferation of both TE-1 and EC-1 cells dependent on time and concentration ( $\mathrm{P}<0.05$, Fig. $2 \mathrm{~A}$ and $\mathrm{B}$ ). In particular, AG490 at $80 \mu \mathrm{mol} / 1$ markedly inhibited cell proliferation compared with the vehicle-treated cells ( $\mathrm{P}<0.001$, Tables I and II).

Additionally, the JAK/STAT3 signaling pathway is known for its regulatory role in the cell cycle. TE-1 cells incubated 

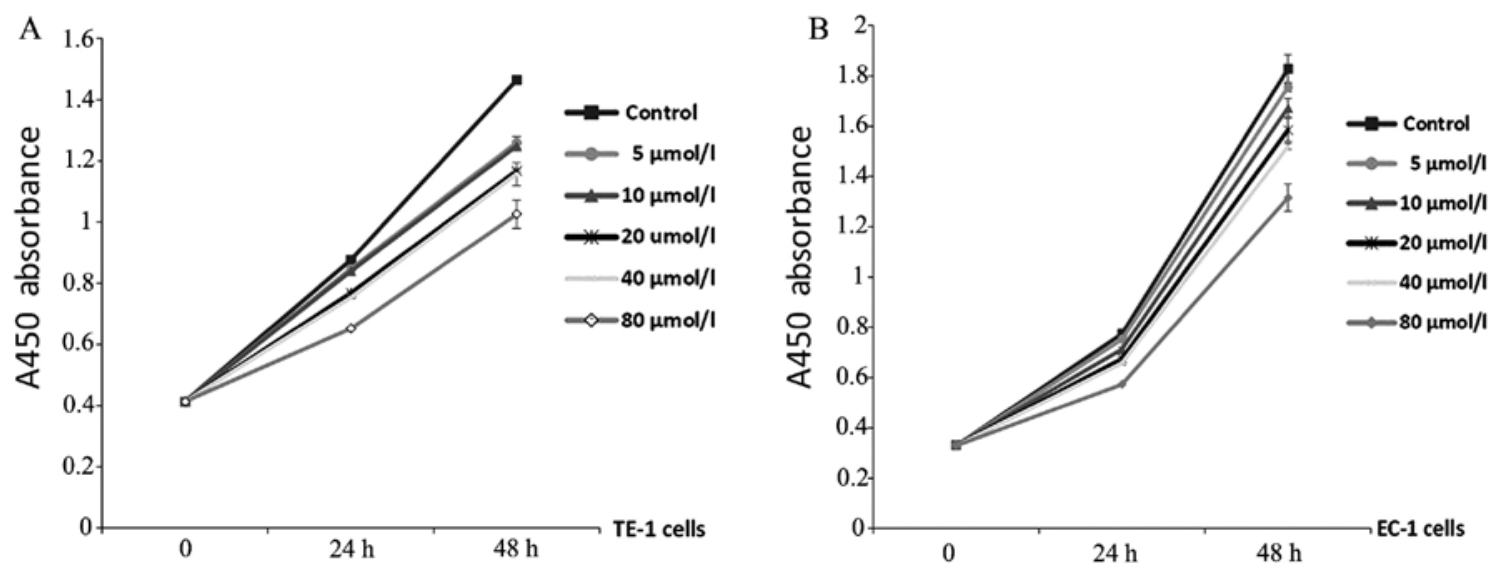

Figure 2. (A) Proliferation curve of control TE-1 cells and cells treated with AG490 at 5, 10, 20,40 and $80 \mu \mathrm{mol} / \mathrm{l}$ for 24 and $48 \mathrm{~h}$, respectively. (B) Proliferation curve of control EC-1 cells and cells treated with AG490 with 5, 10, 20, 40 and $80 \mu \mathrm{mol} / 1$ for 24 and $48 \mathrm{~h}$, respectively. AG490, JAK2 inhibitor.
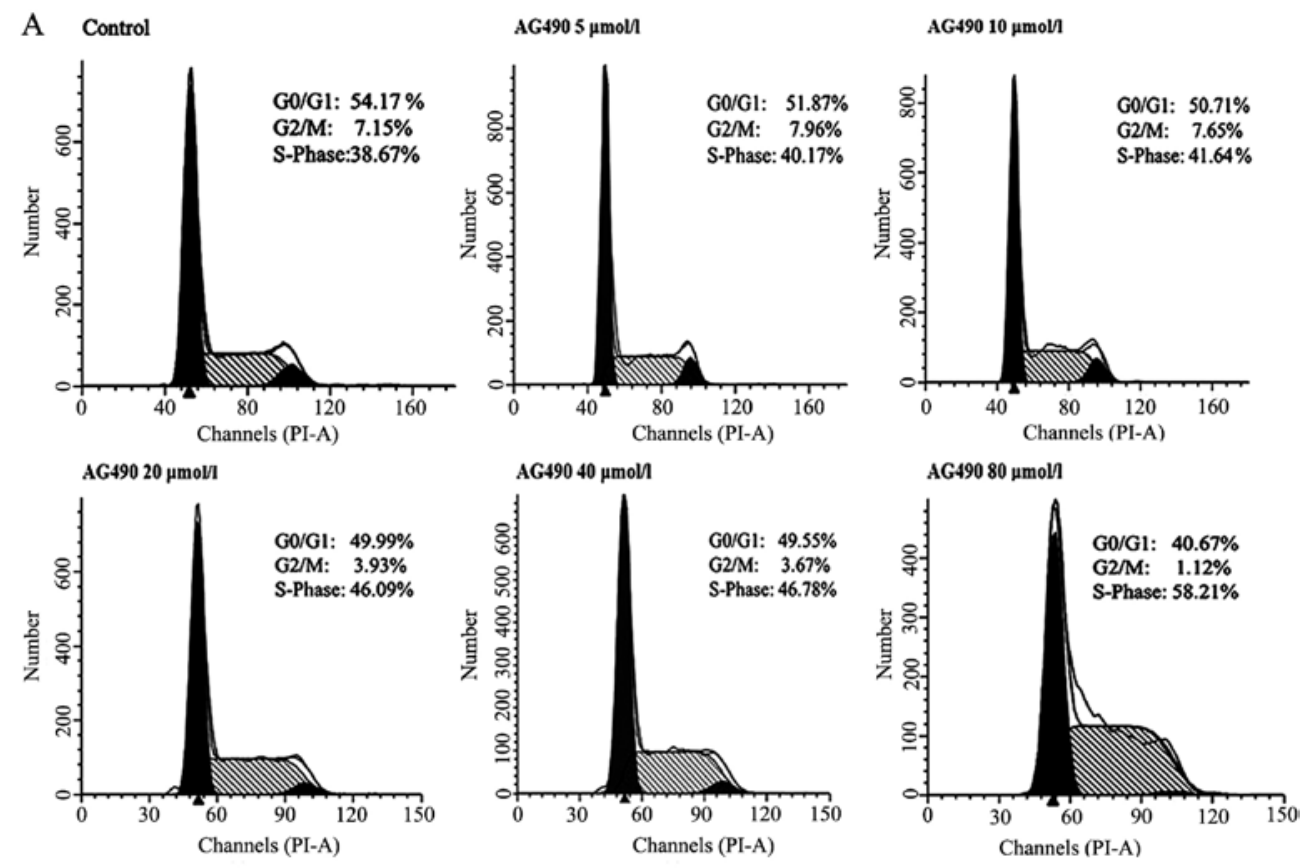

B

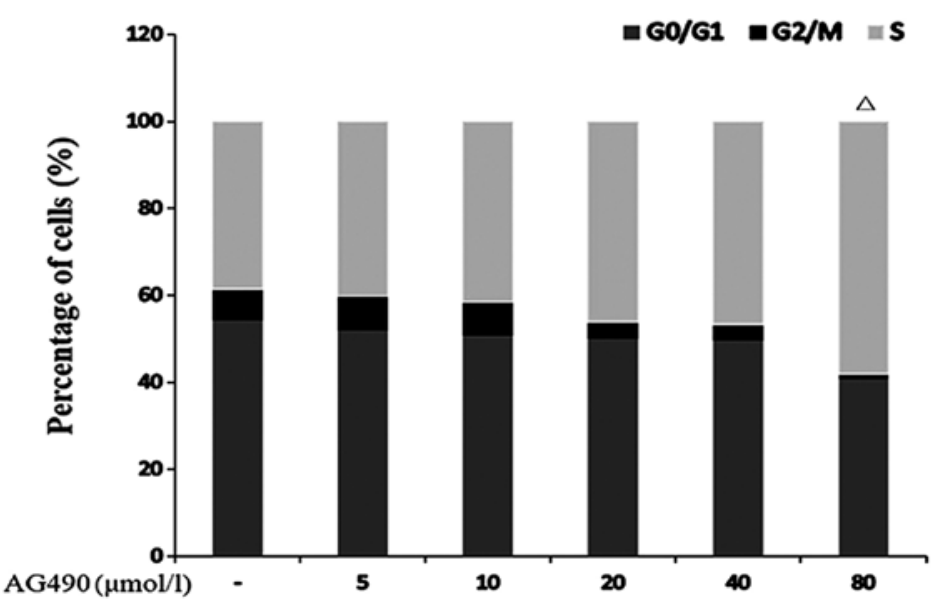

Figure 3. (A) TE-1 cells were treated with AG490 at 5, 10, 20, 40 and $80 \mu \mathrm{mol} / 1$ for $24 \mathrm{~h}$, respectively for flow cytometric analysis. (B) Bars indicate the percentages of cells in the different cell cycles in the control group and the AG490-treated groups. AG490, JAK2 inhibitor.

in AG490 at different concentrations were detected for cell cycle distribution. A decline in the percentage of cells in the
G0/G1 phase and an elevation in the percentage of cells in the $\mathrm{S}$ phase were noted following treatment with increasing 
A
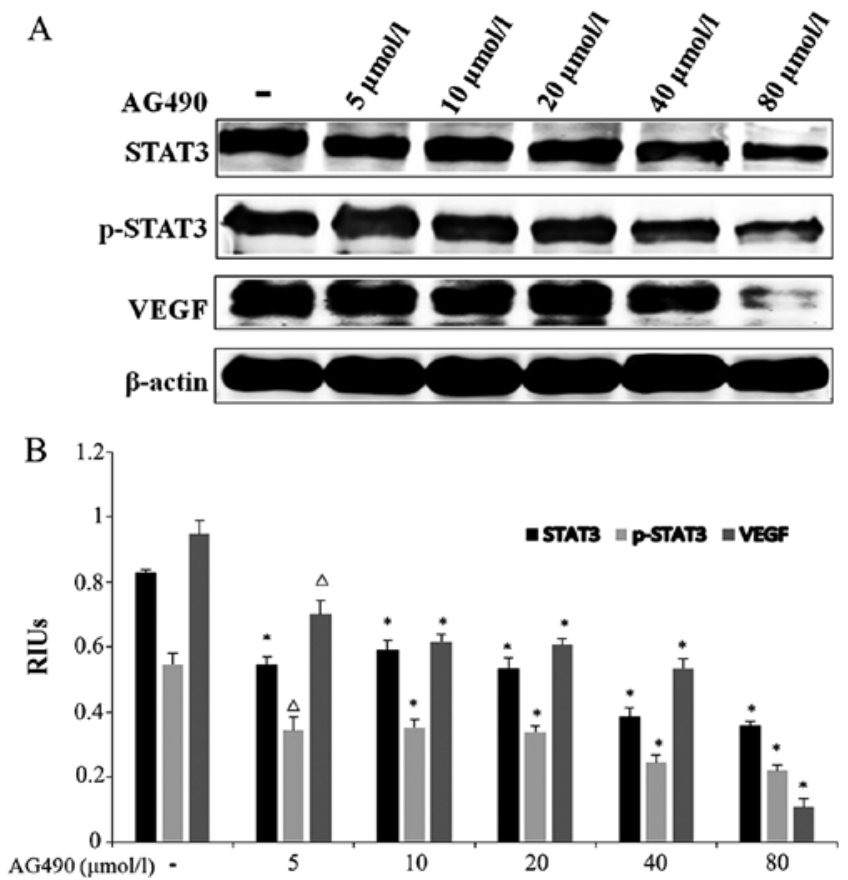

Figure 4. (A) Western blot analysis of STAT3, STAT3 phosphorylation and VEGF protein levels in TE-1 cells treated with or without AG490. (B) Bar graph shows the results of densitometry depicted as mean $( \pm$ SE) relative intensity units (RIUs; normalized to $\beta$-actin) for STAT3, STAT3 phosphorylation and VEGF $\left({ }^{\wedge} \mathrm{P}<0.05,{ }^{*} \mathrm{P}<0.001\right.$ vs. blank control group). STAT3, signal transducer and activator of transcription 3; VEGF, vascular endothelial growth factor.

AG490 concentrations, although only cells treated with $80 \mu \mathrm{mol} / 1$ AG490 achieved statistical significance compared with the vehicle-treated cells $(\mathrm{P}=0.007)$ (Fig. 3A and B).

JAK2 inhibitor inhibits the growth of ESCC by blocking the JAK/STAT3 signaling pathway. AG490, an important JAK2 inhibitor, has been found to exhibit effective inhibition on cancer growth through abrogating the JAK/STAT3 pathway in other cancers. In order to clarify whether it also attenuates ESCC growth by inhibiting the JAK/STAT3 pathway in ESCC, western blotting was conducted to evaluate the protein levels of STAT3 and its phosphorylation levels in TE-1 cells. VEGF, an important factor involved in tumor angiogenesis and metastases regulated by STAT3, was also detected. As illustrated in Fig. 4, the phosphorylated levels of STAT3 and VEGF protein expression decreased in the AG490-treated groups when compared with these levels in the control group; AG490 $80 \mu \mathrm{mol} / 1$ group in particular $(\mathrm{P}<0.001)$. These results indicate that AG490 inhibits ESCC growth and angiogenesis by blocking the JAK/STAT3 pathway.

$J A K 2$ inhibitor inhibits CRI in ESCC through crosstalk between the JAK/STAT3 and NF- $\kappa B$ and COX-2 inflammatory pathways. A potential relationship between CRI and carcinogenesis has been focused on in recent years since various inflammatory factors have been discovered to be involved among which NF- $\mathrm{kB}$ and COX-2 activations have been observed in many types of cancers. However, how the STAT3 pathway interacts with them to promote cancer growth, partic-
A
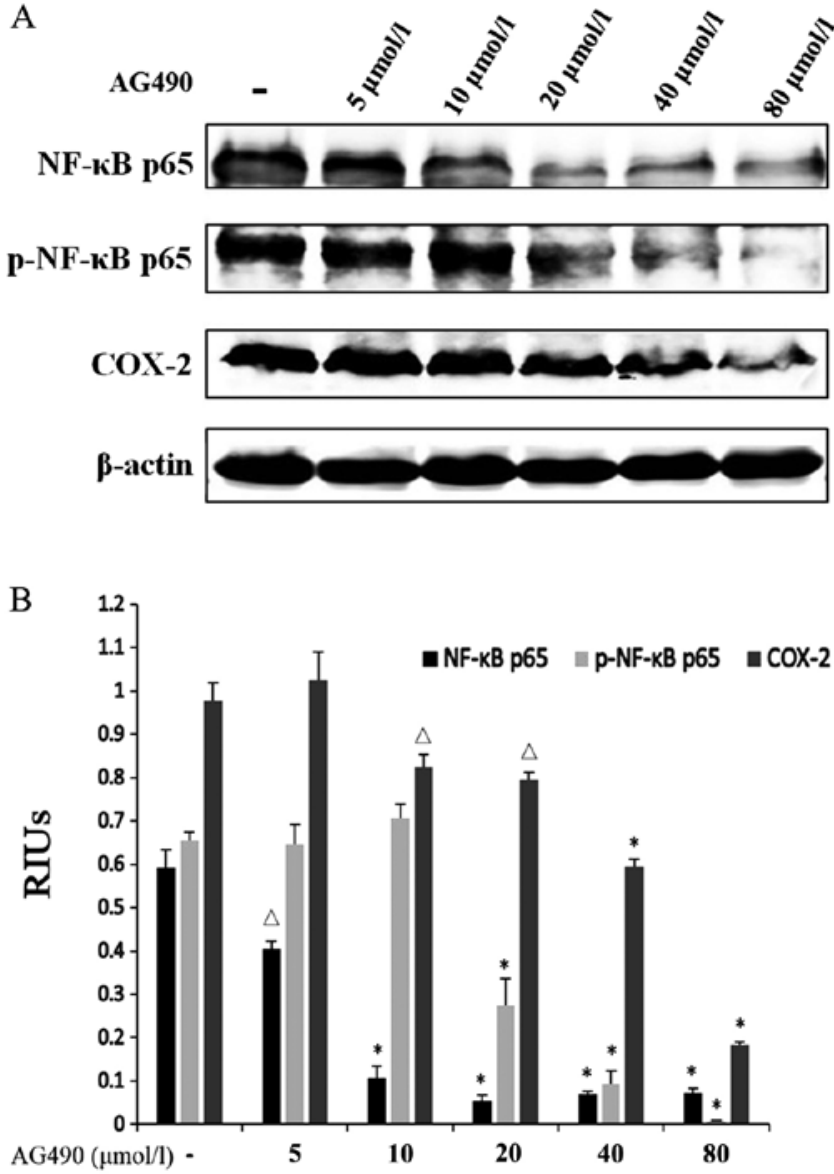

Figure 5. (A) Western blot analysis shows that protein expression of NF- $\kappa B$ p65, NF- $\mathrm{kB}$ p65 phosphorylation, and COX-2 was also decreased in the TE-1 cells after AG490 treatment compared to the control group (B) Bar graph shows the results of densitometry depicted as mean $( \pm \mathrm{SE})$ relative intensity

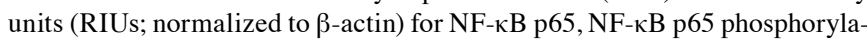
tion and $\mathrm{COX}-2$. NF- $\mathrm{\kappa}$, nuclear factor- $\mathrm{\kappa}$; COX-2, cyclooxygenase-2.

ularly STAT3-COX-2 crosstalk has been poorly studied. In an attempt to shed light on the mechanism of STAT3 signaling pathway in CRI, western blotting was performed to study the crosstalk between STAT3 and NF- $\kappa B$, and COX-2. Since all subunits of NF- $\kappa \mathrm{B}, \mathrm{RelA} / \mathrm{p} 65$ are closely associated with cancer development and inflammation, NF- $\mathrm{kB}$ p 65 was chosen for western blotting. AG490 was also used to treat TE-1 cells to examine the role of JAK/STAT3 in CRI. Protein expressions of NF-kB p65 and its phosphorylated levels and COX-2 were detected following AG490 treatment for $48 \mathrm{~h}$. Expectedly,

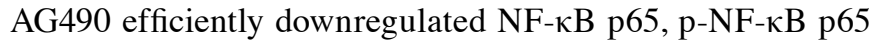
and COX-2 in a concentration-dependent manner (Fig. 5). Our hypothesis that JAK/STAT3 also plays an important role in CRI in ESCC through crosstalk with the NF-кB pathway and COX-2 was confirmed by the above results. Abrogation of the STAT3 signaling pathway also inhibited CRI through downregulation of inflammatory factors NF- $\mathrm{kB}$ and COX-2.

Moreover, we also validated the effects of AG490 on IL-6 with an IL-6 ELISA kit. As a result, reduction in the IL-6 concentration was observed in the TE- 1 cells after being treated with AG490 at 5, 10 and $20 \mu \mathrm{mol} / 1$ for $24 \mathrm{~h}(\mathrm{P}=0.009$, $\mathrm{P}=0.001$ and $\mathrm{P}<0.001$, respectively), and the inhibitory effect was strongest in the AG490 $20 \mu$ mol group. Notably, when the 


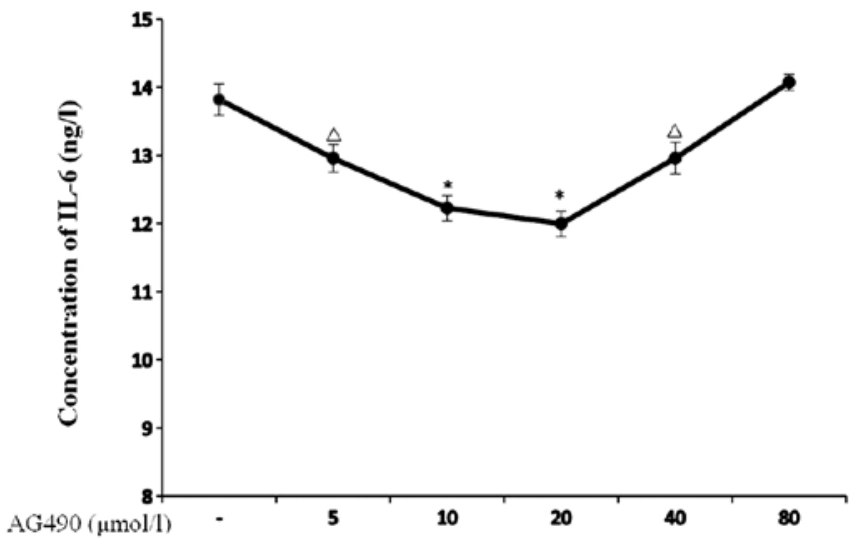

Figure 6. Concentration of IL-6 was tested by an ELISA kit in TE-1 cells after treatment with AG490 at 5, 10, 20, 40 and $80 \mu \mathrm{mol} / 1$ for $48 \mathrm{~h}$, respectively. IL-6, interleukin-6.

AG490 concentration increased to $40 \mu \mathrm{mol} / \mathrm{l}$, the inhibitory effect of AG490 on IL-6 decreased compared to the $20 \mu \mathrm{mol} / 1$ group, although it was still stronger than the control group $(\mathrm{P}=0.01)$. The AG490 $80 \mu \mathrm{mol} / \mathrm{l}$ group did not show any inhibitory effects on IL- 6 when compared with the control group (Fig. 6).

IL- 6 promotes ESCC growth and CRI. As a downstream gene of IL-6, STAT3 activation in tumors can be either IL-6- dependent or -independent. To determine whether STAT3 was activated by IL-6 in ESCC, we performed western blotting. Due to the low basal STAT3 phosphorylation levels noted in our previous data, EC-1 cells were chosen for IL-6 stimulation. IL-6 $(100 \mathrm{ng} / \mathrm{ml})$ treatment for $24 \mathrm{~h}$ increased the phosphorylated STAT3 level and VEGF expression in the EC-1 cells, indicating that the activation of the JAK/STAT3 signaling pathway is IL-6-dependent in ESCC (Fig. 7A and B). Particularly, marked elevation in the p-STAT3 and VEGF protein levels confirmed that IL-6 activated STAT3 and promoted angiogenesis $(\mathrm{P}<0.001)$. Similar results were also found in the NF- $\mathrm{B}$ and COX-2 pathways with the upregulation of NF- $\kappa \mathrm{B}$ p 65 phosphorylation levels and $\mathrm{COX}-2$ protein levels in the EC-1 cells, implying that IL- 6 also induced CRI $(\mathrm{P}<0.05)$ (Fig. 7C and D).

Interaction of STAT3, NF- $\kappa B$ and COX-2 at the mRNA level. To study the mechanism underlying the interaction of STAT3, NF- $\kappa$ B and COX-2, siRNAs of STAT3 and NF- $\kappa$ B were transfected in the TE-1 cells, respectively. Three siRNAs targeted at each gene were designed. siRNAs with the highest inhibition rates were selected for STAT3 and NF- $\kappa \mathrm{B}$ inhibition ( $\mathrm{P}=0.004$ and $\mathrm{P}=0.027$, respectively) (Fig. 8A) to assess the effects of siRNAs on the COX-2 mRNA level. The obvious reduction in COX-2 mRNA expression by both STAT3 and $\mathrm{NF}-\kappa \mathrm{B}$ siRNA suggested that both STAT3 and NF- $\kappa \mathrm{B}$ regulate the transcription of $\mathrm{COX}-2(\mathrm{P}=0.037$ and $\mathrm{P}=0.014$, respectively) (Fig. 8B).
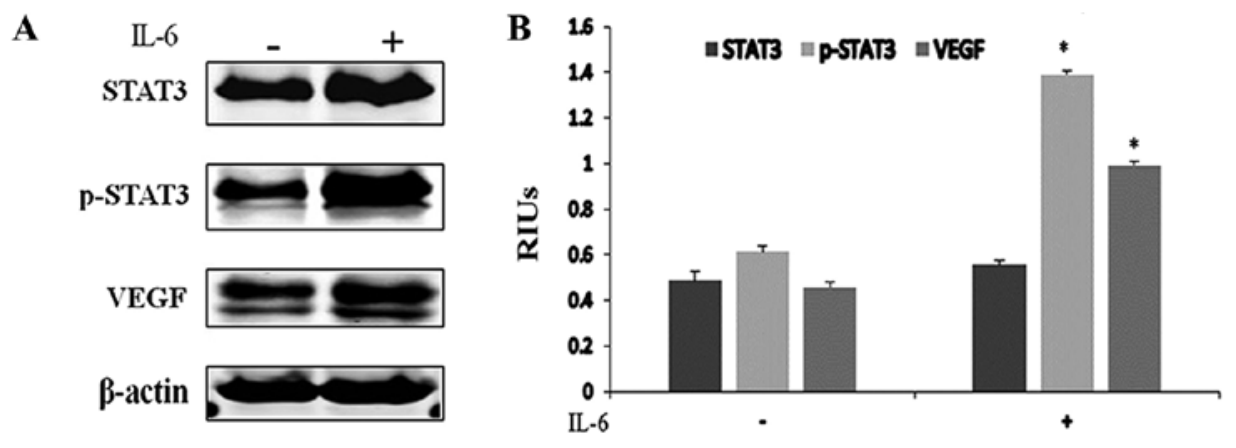

C

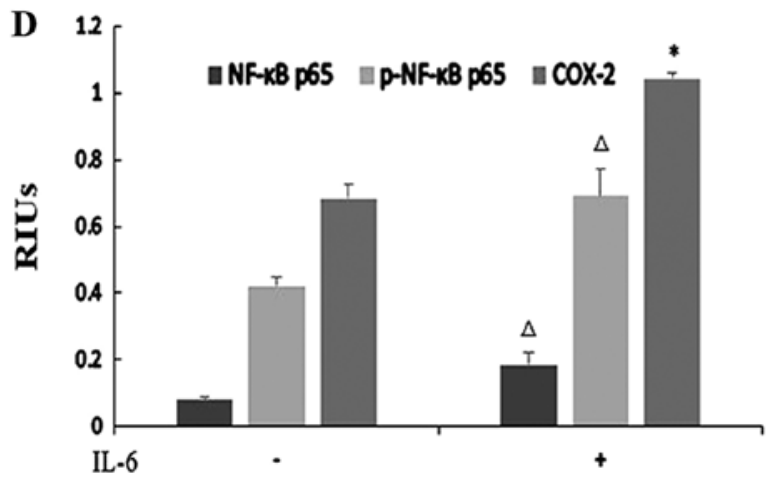

Figure 7. (A) Western blot analysis shows that STAT3 phosphorylation and VEGF protein levels after treatment with IL-6 for $24 \mathrm{~h}$ were markedly elevated in the EC-1 cells compared to the control group while STAT3 expression was slightly increased. (B) Bar graph shows the results of densitometry depicted as mean $( \pm$ SE) relative intensity units (RIUs; normalized to $\beta$-actin) for STAT3, STAT3 phosphorylation and VEGF. ("P<0.001 vs. blank control group) before and after IL-6 stimulation. (C) Western blot results demonstrate that IL-6 also stimulated NF- $\mathrm{kB}$ p65 phosphorylation and increased COX-2 protein levels $(\mathrm{P}<0.05)$. (D) Bar graph shows the results of densitometry depicted as mean $( \pm \mathrm{SE}$ ) relative intensity units (RIUs; normalized to $\beta$-actin) for NF- $\mathrm{kB}$ p65, NF- $\mathrm{\kappa B}$ p65 phosphorylation and COX-2 before and after IL-6 stimulation $\left({ }^{\wedge} \mathrm{P}<0.05,{ }^{*} \mathrm{P}<0.001\right.$ vs. blank control group). STAT3, signal transducer and activator of

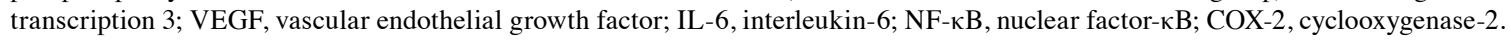



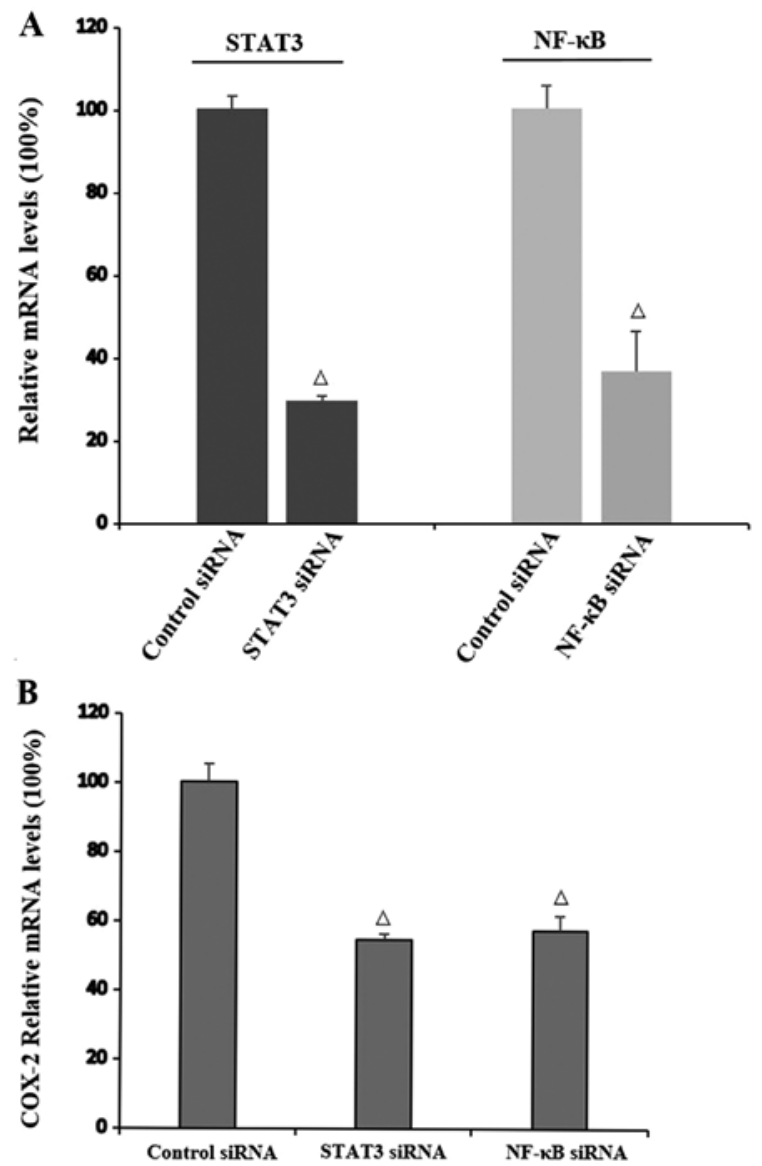

Figure 8. (A) STAT3 and NF- $\kappa$ B siRNA efficiently inhibited the STAT3 and NF- $\kappa B$ mRNA level, respectively. (B) Both STAT3 and NF- $\kappa B$ siRNA decreased the COX-2 mRNA level. STAT3, signal transducer and activator of

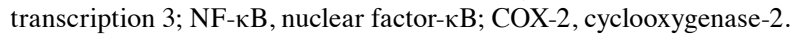

\section{Discussion}

The JAK/STAT3 signaling pathway plays a vital role in tumorigenesis by regulating downstream genes. Activation or phosphorylation of several kinases such as JAK and Src lead to the persistent activation of STAT3 in cancer (23). The availability of AG490, a JAK2 inhibitor, makes it possible to investigate the effect of JAK inhibition on STAT3 activation and the role of the JAK/STAT3 pathway in tumors. In the present study, STAT3 was activated in 3 cell lines at different levels as in many other cancer types. STAT3 phosphorylation was highest in the TE-1 and lowest in the EC-1 cells. After treatment of AG490, cell proliferation was inhibited dependent on AG490 concentration and time both in the TE-1 and EC-1 cells. Experiments focused on the cell cycle demonstrated a decline in cells in the G0/G1 phase and an elevation of cells in the $\mathrm{S}$ and G2/M phases. Although only the AG490 $80 \mu \mathrm{mol} / 1$ group had statistical difference compared to the control group, a regulatory trend of AG490 on TE-1 cells was observed. Additionally, AG490 treatment led to a significant decrease in STAT3 phosphorylation and VEGF protein levels in the TE-1 cells. These results suggest that STAT3 accelerated tumor progression of ESCC by regulating cell growth, cell cycle and angiogenesis. AG490 efficiently blocked tumor growth and progression by suppressing STAT3 activity.

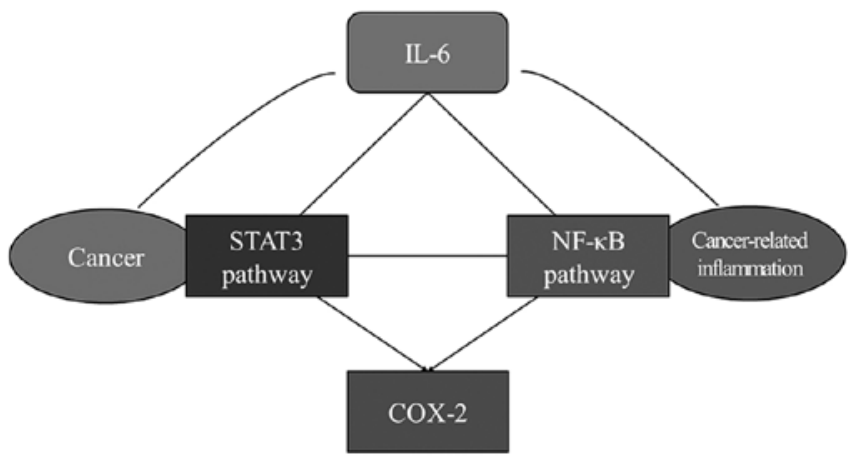

Figure 9. STAT3 signaling pathway links cancer and cancer-related inflammation. STAT3, signal transducer and activator of transcription 3; NF-кB, nuclear factor-кB, IL-6, interleukin-6.

Clinical and epidemiological studies suggest a strong association between chronic infection, inflammation and cancer. Genetic studies reveal that there may be molecular mechanisms linking inflammation and CRI (24). As research indicates the new role of the STAT3 signaling pathway in CRI, we wanted to ascertain whether the JAK/STAT3 pathway plays a central role in regulating cancer growth and CRI in ESCC. NF- $\kappa \mathrm{B}$ is known for the activation in many human cancers and inflammatory processes. The finding that the RelA, encoding p65 subunit of NF- $\kappa B$ is homologous to the viral oncogene $\mathrm{v}-\mathrm{Rel}$ indicates that $\mathrm{NF}-\kappa \mathrm{B}$ is involved in cancer. Recently, the NF- $\mathrm{KB}$ family member, RelA/p65, has been found to physically interact with STAT3 (20). In addition, STAT3 and NF- $\mathrm{kB}$ are both transcriptional factors which play pivotal roles in various aspects of the tumorigenic process by regulating downstream genes. Once activated, NF- $\kappa B$ and STAT3 regulate downstream genes related to cell apoptosis, proliferation and immune response, some of which overlap and require a co-effect of the two factors (25). Recently, it has been found that maintenance of NF- $\mathrm{KB}$ activation in tumors requires STAT3. In the present study, we found that the JAK2 inhibitor also blocked activation of NF- $\mathrm{KB}$ p65, implicating that there is an interaction between STAT3 and NF- $\kappa$ B and that the STAT3 signaling pathway regulates NF- $\mathrm{KB}$ through the p65 subunit. The STAT3 signaling pathway inhibits CRI through interaction with NF- $\mathrm{KB}$ pathway.

In order to examine the role of the STAT3 signaling pathway in CRI, it is essential to study other inflammatory factors closely related to cancer which are also influenced by the STAT3 signaling pathway in addition to NF- $\mathrm{\kappa B}$. COX-2 is an important enzyme which mediates inflammatory processes. Improper upregulation of COX-2 leads to an increase in prostaglandin E2 (PGE2), resulting in pathophysiology of certain types of human cancers as well as inflammatory disorders (26). There is also evidence that COX-2 is overexpressed in esophageal cancer both in ESCC and adenocarcinoma and, inhibition of COX-2 suppresses cancer growth and induces apoptosis $(27,28)$. Nevertheless, studies focused on the STAT3-COX-2 interaction are rare compared to other inflammatory factors. In the present study, STAT3 phosphorylation and COX-2 were co-expressed highly in the ESCC cell lines. The expression levels of the two proteins were consistent in the 3 cell lines, which indicated that the cell line with higher 
p-STAT3 also had higher COX-2 protein levels. AG490 not only efficiently suppressed the STAT3 activation but also decreased COX-2 protein levels significantly. It appeared that COX- 2 could also be affected by the STAT3 pathway. To better clarify the relationship between STAT3, NF- $\mathrm{B}$ and COX-2, we silenced STAT3 and NF- $\kappa$ B by siRNA, respectively. We found at the mRNA levels that both silencing of STAT3 and $\mathrm{NF}-\kappa \mathrm{B}$ downregulated COX-2 mRNA expression, indicating that both STAT3 and NF- $\kappa$ B regulate COX-2. COX-2 may be one of the overlapping downstream genes regulated by STAT3 and $\mathrm{NF}-\kappa \mathrm{B}$.

IL-6 is an important cytokine participating in both inflammation and oncogenesis. When stimulated by IL-6, gp130 is phosphorylated and thereby activates JAK1 and JAK2, leading to the activation of STAT3 (18). However, STAT3 activation in tumors can be either dependent on or independent of IL-6 signaling as mentioned before. To determine whether STAT3 phosphorylation is IL-6-dependent in our ESCC cell lines, we used IL-6 to stimulate EC-1 cells which expressed low STAT3 phophorylation. Analysis of our results revealed that cells treated with IL-6 had significantly increased expression of p-STAT3 and VEGF. Therefore, in ESCC, the aberrant activation of STAT3 was IL-6-dependent, and IL-6 promoted cancer growth and angiogenesis by activating the STAT3 pathway and its downstream genes such as VEGF. In addition, we found that IL- 6 also stimulated NF- $\kappa$ B p 65 activation and upregulated COX-2, implicating that IL- 6 could also stimulate $\mathrm{CRI}$ by activating the NF- $\mathrm{B}$ and COX-2 pathways. Therefore, IL-6 is not only an important cytokine linking the STAT3 and $\mathrm{NF}-\kappa \mathrm{B}$ pathways but is also a bridge through which STAT3 links cancer growth and CRI in ESCC. Moreover, it is necessary for us to ascertain whether attenuating the STAT3 pathway influences the IL- 6 concentration in turn. As a result, AG490 at 5, 10 and $20 \mu \mathrm{mol} / 1$ efficiently reduced the IL-6 concentration in the TE-1 cells, suggesting that blocking the JAK/STAT3 pathway can also decrease IL-6; another way to inhibit CRI. However, notably, when the AG490 concentration increased to 40 and $80 \mu \mathrm{mol} / 1$, the IL- 6 concentration increased compared with the $20 \mu \mathrm{mol} / 1$ group. The probable explanation was that there was a negative feedback in the IL-6-JAK-STAT3 pathway and another possibility was there may be other signaling pathways activated to produce IL-6 when the STAT3 pathway is blocked.

Notably, the inhibitory effects of AG490 on cell proliferation seemed modest compared with other chemotherapeutic agents. It may be more appropriate to conduct AG490 treatment in tumor initiation such as the process from CRI to cancer rather than reducing tumor size based on its suppression of CRI. Animal models are further needed to verify the role of AG490 in the prevention of CRI and cancer growth in ESCC. In conclusion, our research showed that the JAK/STAT3 pathway promoted tumor progression by regulating cell growth, cell cycle and angiogenesis in ESCC. Moreover, STAT3 participated in CRI in ESCC through IL- 6 and interacting with the NF- $\mathrm{NB}$ p65 subunit and COX-2 which are important inflammatory factors accelerating cancer growth (Fig. 9). The JAK/STAT3 pathway is an important pathway which links CRI and cancer growth. The STAT3 pathway could be a novel target both for cancer treatment and prevention in ESCC, and JAK2 inhibitor AG490 could be an option.

\section{Acknowledgements}

This study was supported by the National Natural Science Foundation of China (grant nos. 30872591 and 81372749).

\section{References}

1. Siegel R, Naishadham D and Jemal A: Cancer statistics, 2013. CA Cancer J Clin 63: 11-30, 2013.

2. Wei WQ, Yang J, Zhang SW, Chen WQ and Qiao YL: Analysis of the esophageal cancer mortality in 2004-2005 and its trends during last 30 years in China. Zhonghua Yu Fang Yi Xue Za Zhi 44: 398-402, 2010 (In Chinese).

3. Colotta F, Allavena P, Sica A, Garlanda C and Mantovani A: Cancer-related inflammation, the seventh hallmark of cancer: links to genetic instability. Carcinogenesis 30: 1073-1081, 2009.

4. Suzuk L, Noffsinger AE, Hui YZ and Fenoglio-Preiser CM: Detection of human papillomavirus in esophageal squamous cell carcinoma. Cancer 78: 704-710, 1996.

5. Antunes LC, Prolla JC, de Barros Lopes A, da Rocha MP and Fagundes RB: No evidence of HPV DNA in esophageal squamous cell carcinoma in a population of Southern Brazil. World J Gastroenterol 19: 6598-6603, 2013.

6. Yang GZ, Li L, Ding HY and Zhou JS: Cyclooxygenase-2 is overexpressed in Chinese esophageal squamous cell carcinoma, and

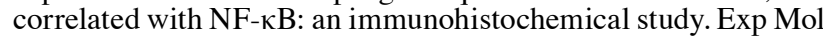
Pathol 79: 214-218, 2005.

7. Mantovani A, Allavena P, Sica A and Balkwill F: Cancer-related inflammation. Nature 454: 436-444, 2008.

8. Bollrath $\mathrm{J}$ and Greten FR: IKK/NF- $\kappa \mathrm{B}$ and STAT3 pathways: central signalling hubs in inflammation-mediated tumour promotion and metastasis. EMBO Rep 10: 1314-1319, 2009.

9. Yu H and Jove R: The STATs of cancer - new molecular targets come of age. Nat Rev Cancer 4: 97-105, 2004.

10. Chung SS, Giehl N, Wu Y and Vadgama JV: STAT3 activation in HER2-overexpressing breast cancer promotes epithelial-mesenchymal transition and cancer stem cell traits. Int J Oncol 44: 403-411, 2014.

11. Qu Y, Oyan AM, Liu R, et al: Generation of prostate tumorinitiating cells is associated with elevation of reactive oxygen species and IL-6/STAT3 signaling. Cancer Res 73: 7090-7100, 2013.

12. Burke WM, Jin X, Lin HJ, et al: Inhibition of constitutively active Stat 3 suppresses growth of human ovarian and breast cancer cells. Oncogene 20: 7925-7934, 2001.

13. Huang C, Cao J, Huang KJ, et al: Inhibition of STAT3 activity with AG490 decreases the invasion of human pancreatic cancer cells in vitro. Cancer Sci 97: 1417-1423, 2006.

14. Xiong H, Zhang ZG, Tian XQ, et al: Inhibition of JAK1, 2/STAT3 signaling induces apoptosis, cell cycle arrest, and reduces tumor cell invasion in colorectal cancer cells. Neoplasia 10: 287-297, 2008.

15. Turkson J and Jove R: STAT proteins: novel molecular targets for cancer drug discovery. Oncogene 19: 6613-6626, 2000.

16. Schindler C and Darnell JE Jr: Transcriptional responses to polypeptide ligands: the JAK-STAT pathway. Annu Rev Biochem 64: 621-652, 1995.

17. Yu H, Pardoll D and Jove R: STATs in cancer inflammation and immunity: a leading role for STAT3. Nat Rev Cancer 9: 798-809, 2009.

18. Park EJ, Lee JH, Yu GY, et al: Dietary and genetic obesity promote liver inflammation and tumorigenesis by enhancing IL-6 and TNF expression. Cell 140: 197-208, 2010.

19. Grivennikov S, Karin E, Terzic J, et al: IL-6 and Stat3 are required for survival of intestinal epithelial cells and development of colitis-associated cancer. Cancer Cell 15: 103-113, 2009.

20. Karin M: Nuclear factor- $\kappa \mathrm{B}$ in cancer development and progression. Nature 441: 431-436, 2006.

21. Greten FR, Eckmann L, Greten TF, et al: IKK $\beta$ links inflammation and tumorigenesis in a mouse model of colitis-associated cancer. Cell 118: 285-296, 2004.

22. He G and Karin M: NF- $\mathrm{kB}$ and STAT3 - key players in liver inflammation and cancer. Cell Res 21: 159-168, 2010.

23. Lo HW, Hsu SC, Xia W, et al: Epidermal growth factor receptor cooperates with signal transducer and activator of transcription 3 to induce epithelial-mesenchymal transition in cancer cells via up-regulation of TWIST gene expression. Cancer Res 67: 9066-9076, 2007. 
24. Coussens LM and Werb Z: Inflammation and cancer. Nature 420 860-867, 2002.

25. Grivennikov SI and Karin M: Dangerous liaisons: STAT3 and $\mathrm{NF}-\kappa \mathrm{B}$ collaboration and crosstalk in cancer. Cytokine Growth Factor Rev 21: 11-19, 2010.

26. Hao Q, Zhang $\mathrm{C}$, Gao Y, et al: FOXP3 inhibits NF- $\kappa \mathrm{B}$ activity and hence COX2 expression in gastric cancer cells. Cell Signal 26: 564-569, 2014
27. von Rahden BH, Stein HJ, Pühringer F, et al: Coexpression of cyclooxygenases (COX-1, COX-2) and vascular endothelial growth factors (VEGF-A, VEGF-C) in esophageal adenocarcinoma. Cancer Res 65: 5038-5044, 2005.

28. Zhi H, Wang L, Zhang J, et al: Significance of COX-2 expression in human esophageal squamous cell carcinoma. Carcinogenesis 27: 1214-1221, 2006. 